\title{
La mirada desde la periferia: desarrollos en la historia de la frontera colombiana, desde 1970 hasta el presente
}

\author{
Jane M. Rausch \\ University of Massachusetts-Amherst (Estados Unidos) \\ jrausch@history.umass.edu \\ Traducción de \\ Juan Pablo Fernández

\section{Resumen $^{1}$}

En este artículo se analizan tres cambios fundamentales que han ocurrido en el estudio de las fronteras en Colombia entre 1970 y 2003. Entre los temas de estudio se encuentran la transformación de la "frontera" como construcción histórica; el incremento en el número de estudios académicos acerca de la historia y la cultura de la frontera colombiana; y, finalmente, la integración de estos nuevos estudios de la frontera con el grueso de la historiografía colombiana. La autora concluye señalando que estas tendencias actuales seguirán en auge y deberán contribuir al mejor entendimiento del pasado colombiano.

Palabras clave: HISTORIOGRAFÍA, TENDENCIAS, FRONTERAS, COLOMBIA.

\begin{abstract}
This article will analyze three fundamental changes which took place in the study of the Colombian borders from 1970 to 2003. Among the themes of study are the transformation of the notion of 'border' as a historical construction; the increase in the number of academic studies treating the history and the culture of formation of the Colombian borders; and, finally, the integration of these new studies about border with the body of Colombian historiography. The author's conclusion suggests that the current tendencies continue to be in vogue, and will contribute to a better understanding of the Colombian past.
\end{abstract}

Key words: HISTORIOGRAPHY, TENDENCIES, BORDERS, COLOMBIA.

\footnotetext{
${ }^{1}$ Una versión preliminar de este texto fue presentada como ponencia en el XIII Congreso de Colombianistas, agosto 12 a 15 de 2003, Barranquilla, Colombia.
} 
Hace casi cuarenta años, cuando participaba en un seminario para estudiantes de posgrado patrocinado por la University of Wisconsin y el Land Tenure Center, llegué a Barranquilla como parte de mi primer viaje a Colombia. A pesar de que mi grupo rápidamente se desplazó a Bogotá, donde emprendí un estudio de la educación primaria en Colombia durante la época del Federalismo, jamás he olvidado las primeras impresiones que tuve de Barranquilla y de Santa Marta, la cual en 1964 era todavía un pueblo apacible a donde no habían llegado los rascacielos y hoteles que hoy rodean sus hermosas playas. Ese mismo verano, y partiendo de Bogotá, visité por primera vez los Llanos del Meta. El viaje por carretera entre Bogotá y Villavicencio fue una experiencia emocionante, especialmente para alguien como yo que creció en una zona tan plana como el "Midwest" de Estados Unidos. Al llegar a Vistahermosa, el punto donde las montañas se hacen a un lado y por primera vez dejan ver al viajero las inmensas llanuras, sentí la misma euforia que sintió el alemán Ernst Röthlisberger cuando llegó a este mismo punto en 1882. El escribió:

¿Cómo describir nuestro asombro y nuestra delicia al ver extendida súbitamente ante nosotros la inmensidad de los Llanos? No se podría imaginar contraste más impresionante y fuerte que el que forman las macizas, inextricables cordilleras, que ascienden hasta la región de las nieves perpetuas, y esta uniforme llanura tropical [...]. Los Llanos tienen movimientos de color y diversidad sin fin; son una imagen de la vida, que no predica al hombre su total impotencia, sino que, al menos, despierta en él esperanzas como las que se alzaron entre los compañeros de Colón al escuchar el mágico ¡Tierra! ¡Tierra! ${ }^{2}$.

Esta imagen de los Llanos como tierra de promisión —o, como a veces son llamados, "el futuro de Colombia"- me quedó firmemente grabada, tanto que, cuando volví a Colombia en 1973, decidí escribir una historia de los Llanos del Meta, el Casanare y Arauca. No quise escribir apenas una historia de la región, sino que la quise enfocar desde el punto de vista de la idea de "frontera", la cual, en el sentido que hace ya más de cien años le dio Frederick Turner, ha desempeñado un papel único en el desarrollo de Colombia como nación. Treinta años y tres libros después, mi entusiasmo por este tema sigue siendo tan intenso como antes; sin embargo, con el paso del tiempo se han presentado cambios dramáticos: primero, en mi propia comprensión de la frontera como construcción histórica; segundo, en el estudio de las regiones fronterizas colombianas en general; $y$, finalmente, en el impacto de los estudios de la frontera en la historiografía colombiana. Estos tres desarrollos son lo que quiero discutir el día de hoy.

\section{Cambios en la idea de la frontera como construcción histórica}

Cuando comencé a investigar los Llanos en la década de los 70, los historiadores estaban empezando a tener reparos serios acerca de la "tesis de la frontera" desarrollada por Frederick Jackson Turner en su importante trabajo "The Significance of the Frontier in American History" ("El significado de la frontera en la historia de Estados Unidos"), presentado ante la American

\footnotetext{
${ }^{2}$ Ernst Röthlisberger, El Dorado (Bogotá: Banco de la República, 1963), 228 y 229.
} 
Historical Association en su congreso de 1893. En resumen, Turner argüía que la "frontera" norteamericana era conceptualmente diferente de la idea europea de frontera: una línea fronteriza fortificada tendida a través de una zona densamente poblada. En su opinión, el aspecto más importante de la frontera era que ésta se encontraba más allá de la tierra libre. La frontera en Norteamérica no era una línea sino una región que además comprendía el territorio indígena y la margen externa de las "zonas habitadas" reconocidas por los informes de los censos. Turner sostenía que el desarrollo de los Estados Unidos fue anormal en cuanto el país siempre estuvo condicionado por una serie de distintas zonas fronterizas. Gracias a la presencia de enormes extensiones de tierra libre y arable, la frontera en expansión fue una fuerza omnipresente en la historia estadounidense. A esta secuencia de territorios vírgenes fueron llegando olas de pioneros, en un proceso que sutil pero constantemente influenció todos los aspectos de la vida en los Estados Unidos. La experiencia compartida de la vida en la frontera, concluía Turner, hizo de los estadounidenses un pueblo particularmente individualista, democrático y emprendedor ${ }^{3}$.

Casi inmediatamente después de que Turner presentó su tesis, la comunidad académica comenzó a debatir mucho de lo que Turner había querido demostrar pero continuó examinando las posibilidades de su teoría al estudiar otros países donde la "frontera" había sido característica prominente ${ }^{4}$. De este modo, en mi primer libro decidí modificar el concepto afirmando que, para mis propósitos, la frontera colombiana fue una línea en la que la colonización hispánica se encontró con la selva, aunque en este caso la selva no era completamente "salvaje" sino que estaba poblada por comunidades indígenas. Así, la frontera también se constituyó en una zona de interpenetración de dos sociedades hasta ese momento completamente distintas. Con el correr de los siglos la dinámica de interacción entre culturas y gentes en estas zonas produjo una identidad regional que a su vez impactó la metrópoli. Si bien reconocí que la contribución indígena es componente fundamental de esta historia, decidí, dada la naturaleza de las fuentes disponibles, concentrarme en el lado español de la frontera, y en los tres volúmenes que he escrito sobre los Llanos he hecho hincapié en las políticas de los gobiernos español y colombiano y en el impacto que han tenido misioneros, municipios, terratenientes y llaneros en la formación de la frontera, dejando de lado el papel desempeñado por las comunidades indígenas 5 .

Esta aproximación es claramente insostenible en 2003. Otros historiadores y antropólogos han demostrado que la influencia nativa en el desarrollo de la sociedad fronteriza fue como mínimo igual de importante que la influencia colombiana. Para citar apenas un ejemplo, la comunidad académica contemporánea dejó de considerar a los misioneros como figuras heroicas que, expuestas a inmensas amenazas, desafiaron mortales enfermedades tropicales y pacientemente

\footnotetext{
${ }^{3}$ Frederick Jackson Turner, "The Significance of the Frontier in American History", Annual Report of the American Historical Association, 1893 (Washington, D.C.: Government Printing Office, 1894).

${ }^{4}$ Consultar por ejemplo la obra de W. D. Wyman y C. B. Kroeber, The Frontier in Perspective (Madison: University of Wisconsin Press, 1957).

${ }^{5}$ Jane M. Rausch, A Tropical Plains Frontier: The Llanos of Colombia: 1531-1831 (Albuquerque: University of New Mexico Press, 1984), xiv.
} 
se esforzaron en aprender los idiomas nativos con el fin de evangelizar e hispanizar a los "salvajes primitivos". Al contrario: en su importante ensayo "Reflections on the Ibero-American Frontier Mission as an Institution in Native American History" ("Reflexiones sobre la misión fronteriza iberoamericana como institución en la historia nativa americana"), David Sweet ha demostrado que, cuando fueron forzados a aceptar a los misioneros europeos, los indígenas actuaron en interés propio: para evitar ser castigados, para ganar beneficios espirituales o para adquirir objetos importados de Europa. Estos "beneficios" tuvieron su precio, ya que entre lo que vino de Europa se encontraban nuevas enfermedades mortales y un proceso no deseado de desculturización. En vez de asumir pasivamente el papel de "hijos" de los "padres misioneros", los indígenas, según Sweet, siempre opusieron resistencia contra los misioneros y con frecuencia se rebelaron ${ }^{6}$.

Esta visión contemporánea de los misioneros está reflejada en una tesis doctoral recientemente sustentada en la University of Massachusetts. En "Trade and Conversion: Indians, Franciscans and Spaniards on the Upper Amazon Frontier, 1693-1790" ("Comercio y conversión: Indígenas, franciscanos y españoles en la frontera del alto Amazonas, 1693-1790”), Richard Goulet ha demostrado que, cuando se adentraron en la frontera del alto Amazonas en las regiones de Putumayo y Caquetá en el siglo XVIII, los franciscanos, lejos de ser figuras dominantes en la conversión de grupos nativos, tuvieron que introducirse por la fuerza en una red regional de comercio que había existido durante siglos pero que se vio afectada de manera fundamental con la llegada de los europeos y los frailes. Distintas alianzas comerciales y militares entre los indígenas y los europeos produjeron una situación dinámica en la que los franciscanos tuvieron a veces más y a veces menos éxito, pero en 1721 y luego en 1790 los misioneros fueron expulsados violentamente por las mismas comunidades indígenas que estaban intentando evangelizar ${ }^{7}$.

Treinta años de estudio me han hecho modificar mi punto de vista acerca de la frontera colombiana en otros dos aspectos. En primer lugar, poco a poco me fui dando cuenta de que, a diferencia de la frontera estadounidense estudiada por Turner, en la que los europeos se fueron trasladando inexorablemente de oriente a occidente, en los Llanos los europeos fueron llegando por dos flancos. Los territorios del Meta, Arauca y Casanare fueron conquistados y colonizados no solamente por colombianos de la zona montañosa descendiendo del altiplano sino también por alemanes y españoles procedentes de las llanuras y los Andes venezolanos. Cuando empecé a escribir el Volumen II, el cual narra la historia de los Llanos entre 1830 y 1930, fue para mí bastante claro que, especialmente en el territorio de Arauca, fueron los venezolanos, mucho más que los colombianos, quienes dominaron la naciente cultura llanera. En segundo lugar, cuando emprendí la redacción del Volumen III, Colombia: Territorial Rule and the Llanos Frontier

\footnotetext{
${ }^{6}$ David Sweet, "The Ibero-American Frontier Mission in Native American History" en Erick Langer y Robert H. Jackson, ed., The New Latin American Mission History (Lincoln y Londres: University of Nebraska Press, 1995), 148.

${ }^{7}$ Richard James Goulet, "Trade and Conversion: Indians, Franciscans and Spaniards on the Upper Amazon Frontier, 1693-1790", tesis presentada para obtener el título de doctor (Massachusetts: University of Massachusetts-Amherst, 2003).
} 
(Colombia: el gobierno territorial y la región fronteriza de los Llanos), había al fin llegado a entender que Colombia es una tierra de múltiples fronteras: no sólo fronteras internas (como aquélla entre Caldas y Antioquia) sino también fronteras externas constituidas por otras regiones periféricas de las tierras bajas: la Costa Pacífica, las islas de San Andrés y Providencia, y especialmente la Amazonía. Cada una de estas zonas tiene sus características únicas, pero en general todas son regiones cuya integración al resto de la nación se vio aplazada y en las que, con excepción de San Andrés y Providencia, la interacción de los nativos con los europeos, y en el caso de la Costa Pacífica con afrocolombianos, fue el tema dominante en la formación de la sociedad y la cultura fronterizas.

En particular, la región de la Amazonía ha sido objeto de algunos estudios de excelente calidad: El grueso volumen Colombia amazónica (Bogotá: Fondo FEN, 1987) contiene informativos ensayos de investigadores activos como Camilo Domínguez, Héctor Llanos Vargas, Roberto Pineda Camacho y Myriam Jimeno Santoyo; Los pobladores de la selva: Historia de la colonización del noroccidente de la Amazonía, con edición a cargo de Bernardo Tovar Zambrano, es el producto de un esfuerzo colectivo del Instituto Colombiano de Antropología, el Instituto Colombiano de Cultura (hoy Ministerio de Cultura), el Plan Nacional de Rehabilitación (PNR) y la Universidad de la Amazonía. El hecho de que la región de la Amazonia pertenece a varios países ha estimulado diversos esfuerzos de cooperación internacional en estas áreas de investigación. Durante la preparación de su soberbio estudio Red Rubber, Bleeding Trees: Violence, Slavery and Empire in Northwest Amazonia, 1850-1933 (Caucho rojo, árboles sangrando: Violencia, esclavitud e imperio en el noroccidente de la Amazonia, 1850-1933) (Albuquerque: University of New Mexico Press, 1998), Michael Standfield tuvo la oportunidad de consultar archivos y expertos en Perú, Ecuador y Colombia y pudo visitar sitios clave en el Putumayo y en Iquitos.

\section{Desarrollos en el estudio de los Llanos colombianos}

Cuando empecé mis estudios, en 1973, la región de los Llanos había sido, salvo en un par de casos excepcionales, olvidada por los historiadores". Un repaso a la extensa "Guía bibliográfica para los Llanos Orientales de Colombia" recopilada por María Teresa Cobos en 1965 reveló que las investigaciones más importantes habían sido realizadas por geógrafos y antropólogos y que estos académicos con frecuencia lamentaban que la falta de información histórica confiable acerca de las llanuras interponía obstáculos a la producción de documentación científica ${ }^{9}$. A estas quejas académicas deben añadirse los ruegos de gobernantes y habitantes llaneros que, en los años posteriores a la época de La Violencia, pidieron al gobierno nacional que patrocinara

\footnotetext{
${ }^{8}$ Entre las obras más útiles debidas a historiadores podemos mencionar las siguientes: Juan M. Pacheco, Los jesuitas en Colombia (Bogotá: Editorial "San Juan Eudes”, 1959-1962); E. Ortega Ricaurte, Villavicencio (1842-1942): Monografía histórica (Bogotá, 1943) y Raquel Ángel de Flórez, Conozcamos al Departamento del Meta. 2 vols. (Bogotá: : Talleres Gráficos del Fondo Rotatorio Judicial Penitenciaría Central, 1963).

9 María Teresa Cobos, "Guía bibliográfica para los Llanos Orientales de Colombia", Boletín Cultural y Bibliográfico 8, no. 12 (1965): 1888-1935.
} 
estudios sobre la vida en los Llanos para que el pueblo colombiano tuviera una mejor idea de la verdadera cara de esta olvidada región de su país ${ }^{10}$.

Mi primer libro sobre la historia de los Llanos apareció en 1983. Durante su redacción mantuve correspondencia con varios colombianos y venezolanos que se encontraban estudiando distintos aspectos de la historia llanera. Entre estas personas se destacaba María Eugenia Romero, quien en compañía de su hermana, Claudia Romero Moreno, fundó el instituto Orinoquia Siglo XXI y publicó Desde el Orinoco hacia el siglo XXI: El hombre, la fauna y su medio ${ }^{11}$. María Eugenia y yo tuvimos la idea de organizar un simposio que reuniera a académicos colombianos y venezolanos de varias disciplinas con el fin de compartir sus trabajos de investigación en diversos aspectos de la historia de los Llanos. En gran medida debido a la considerable habilidad organizativa de María Eugenia y al respaldo de la Universidad Tecnológica de los Llanos Orientales y la Academia de Historia del Meta, el primer Simposio Internacional de Historia de los Llanos Colombo-Venezolanos tuvo lugar en Villavicencio entre el 11 y el 13 de agosto de 1988. Treinta investigadores en representación de universidades e institutos de Bogotá, Tunja, Yopal, Villavicencio, Cali, Florencia, Arauca, Barcelona (España), Guárico (Venezuela) y Estados Unidos presentaron ponencias durante el simposio, en el cual participaron más de cien personas ${ }^{12}$. El éxito de esta primera reunión de "llanerólogos" estimuló a los organizadores a seguir realizando el simposio cada dos años. Como resultado, y a pesar de la violencia de que ha sido presa la región en las últimas décadas, se han realizado siete simposios más; el más reciente de ellos en San Carlos de Austria, Cojedes, Venezuela, en septiembre de $2001^{13}$.

Los simposios son sólo una parte de la nueva ola de investigación acerca de los Llanos que han emprendido jóvenes académicos con entrenamiento profesional. En Yopal, Casanare, Héctor Publio Pérez, Máster en Historia de la Universidad Pedagógica y Tecnológica de Colombia (UPTC), organizó en 1987 el Centro de Historia del Casanare, el cual publica la revista Caribabare y se esfuerza por preservar los archivos de la región de los efectos nocivos del clima y la negligencia. Pérez, autor de La participación de Casanare en la Guerra de Independencia 1809-1819 14 , ha promovido dos discos compactos titulados "Raíces de la música llanera en Casanare" en los que se pretende preservar el folclor auténtico del Casanare de cara a la creciente modernización que ha traído consigo la explotación petrolífera en la región. Para no

\footnotetext{
${ }^{10}$ Tenemos el ejemplo de Guillermo Ramírez, que en su ensayo "San Luis de Palenque: El llanero y su presente", Económica Colombiana, no. 2 (1954): 21-38, escribió: "Nuestra nación es dada para exagerar o menospreciar nuestros recursos y en el caso de los Llanos se ha olvidado fácilmente la paciente obra de acondicionamiento del hombre a su medio. Estudiar al llanero, su índole, tradiciones, su folclor: desentrañar el hondo significado de sus creencias y supersticiones; mejorar su salud y desarrollar su mente; orientar vocacionalmente a las actividades creadoras congénitamente aptas para el Llano: son imperativos de Gobierno".

${ }^{11}$ María Eugenia Romero, Desde el Orinoco hacia el siglo XXI: El hombre, la fauna y su medio (Bogotá: Fondo FEN, 1989).

${ }^{12}$ Las ponencias fueron recopiladas en el libro Academia del Meta, comp., Los Llanos: Una historia sin fronteras (Bogotá: Crear Arte, 1988).

${ }^{13}$ Los demás fueron realizados en Yopal (1990), Arauca (1992), San Martín, Meta (1995) y San Fernando de Apure, Venezuela (1999).

${ }^{14}$ Héctor Pérez, La participación de Casanare en la Guerra de Independencia 1809-1819 (Bogotá: ABC, 1988).
} 
quedarse atrás, la Asociación Cravo Norte de Arauca organizó en agosto de 1987 el Primer Encuentro Colombo-Venezolano de Escritores Llaneros, cuyas memorias se publicaron bajo el título Sobre los llanos [...] (Bogotá, 1988). Con el respaldo financiero de la Occidental Petroleum, la Asociación ha seguido publicando estudios relacionados con la historia de los Llanos. El musicólogo araucano Miguel Ángel Martín Salazar fundó la Academia Folclórica de Música del Meta, la cual organiza cada año un festival de canciones colombianas y un concurso internacional de joropo. Martín Salazar también es autor de la importante monografía $\mathrm{Del}$ folclor llanero (Bogotá: Editorial Presencia, 1991), cuya nueva edición es solamente uno de los múltiples proyectos que ha patrocinado la Academia de Historia del Meta.

El gobierno colombiano también se ha mostrado interesado en recuperar la historia de los Llanos. En 1991 COLCIENCIAS patrocinó programas para elaborar índices de los archivos municipales de Villavicencio, Restrepo, Cumaral, Granada y San Martín y para crear un archivo histórico para el Departamento del Meta. Además, el establecimiento de programas de posgrado en historia y sociología en varias universidades colombianas ha dado como resultado la aparición de algunas excelentes tesis de maestría sobre distintos aspectos de la región llanera. Dos ejemplos que vale la pena resaltar son "Departamento del Meta: Historia de su integración a la nación, 1536-1936", presentada por Omar Baquero al Departamento de Sociología de la Universidad Nacional de Bogotá, y "Vichada, del Orinoco indígena a la colonización y Marandúa", presentada por Carlos Munar de la UPTC en la década de los 80. En esta categoría podemos también incluir Un pueblo de frontera: Villavicencio 1840-1940 de Miguel García Bustamante (Bogotá: Caragraphics, 1997), quien obtuvo su maestría en la Universidad Nacional Autónoma de México y su doctorado en la Universidad de Sevilla.

\section{El impacto de los estudios de la frontera en la historiografía colombiana}

En 1987, con ocasión de mi aceptación como miembro extranjero correspondiente de la Academia de Historia del Meta, presenté una ponencia titulada "Región olvidada: Los Llanos Orientales en la historia de Colombia". En dicho trabajo di cuenta de una curiosa contradicción: con frecuencia los académicos colombianos mencionan el regionalismo como factor fundamental en el desarrollo histórico de la nación y de inmediato olvidan por completo las dos regiones más extensas (la Amazonia y los Llanos) para concentrarse en la interacción entre las provincias de la cordillera y la Costa Atlántica. Por ejemplo, en la introducción a su historia de la Colombia precolombina, el arqueólogo Gerardo Reichel-Dolmatoff afirma:

Al oriente de los Andes y más allá de la cordillera se extienden inmensas áreas periféricas - las llanuras de la Orinoquia y las selvas de la Amazonia- que constituyen las dos terceras partes del territorio nacional. Estas zonas remotas y escasamente pobladas nunca han desempeñado un papel importante en el desarrollo cultural del país, cuyo centro siempre han sido las cuestas y valles de las cordilleras y las llanuras de aluvión y las zonas costeras de los litorales ${ }^{15}$.

\footnotetext{
${ }^{15}$ Gerardo Reichel-Dolmatoff, Colombia (Nueva York: Thames and Hudson, 1965), 29. Jaime Jaramillo Uribe hizo una afirmación semejante en su ensayo "Algunos aspectos de la personalidad histórica de Colombia" cuando
} 
Naturalmente, en mi ponencia intenté mostrar que ésta era una suposición equivocada: hice hincapié en la poco conocida pero importante participación de los Llanos en la conquista de la Nueva Granada, en la vida económica y religiosa durante el período de la Colonia, en la Revolución de los Comuneros, en la Guerra de Independencia, en los esfuerzos para poblar los Llanos llevados a cabo durante la era de los Estados Unidos de Colombia (1863-1886), en la Guerra de los Mil Días, en la "Revolución en Marcha" de Alfonso López Pumarejo y, por supuesto, en el importante papel desempeñado por los Llanos en la primera ola de La Violencia que siguió al asesinato de Gaitán en 1948. Confieso que me sentí bastante satisfecha cuando descubrí que, en su ensayo "De Carlos E. Restrepo a Marco Fidel Suárez: Republicanismo y gobiernos Conservadores", incluido en la colección Nueva historia de Colombia que editó Álvaro Tirado Mejía (ocho volúmenes, Bogotá: Planeta, 1989), Jorge Orlando Melo incluyó un recuento de la rebelión de Humberto Gómez en Arauca, evento del que veinte años antes quizá sólo los araucanos estaban enterados ${ }^{16}$.

Sin embargo, la historiografía tradicional colombiana ha venido comenzando a ocuparse de regiones que hasta hace poco había tenido olvidadas. Este cambio de actitud bien puede haberse debido a los aún recurrentes fenómenos de la guerrilla y el narcotráfico y sus efectos en estas regiones periféricas, o bien puede ser consecuencia de la información brindada por los fundamentales estudios arriba mencionados; en cualquier caso, se trata de un cambio bienvenido. Así como la Constitución de 1991 elevó estos territorios al rango de Departamentos, los historiadores colombianos están empezando a apreciar el papel decisivo que han desempeñado las regiones más apartadas de la nación. Tras reseñar 426 libros y 38 revistas académicas publicados en Colombia entre 1990 y 2001 y producidos en su mayoría por autores colombianos, James D. Henderson encontró que, con un total de 100, los estudios históricos y materiales relacionados constituyen la segunda categoría más común de escritos (la primera la conforman 126 libros acerca del crimen y la violencia). De estos 100 volúmenes, 25 son estudios regionales y ocho tratan específicamente las zonas fronterizas. Respecto de esta subcategoría, Henderson concluyó que "las historias regionales y estudios sobre regiones fronterizas figuran de manera destacada en la investigación académica emprendida recientemente en Colombia". Refiriéndose a los trabajos de Elsy Marulanda sobre el Sumapaz y a las entrevistas realizadas a colonos por Alfredo Molano y Graciela Uribe Ramón, Henderson añade que "la frontera colombiana es una importante y popular área de investigación""

escribió: "El país se formó y pobló en los Andes y sus alrededores; los Llanos y la sociedad ganadera han desempeñado un papel casi nulo en su desarrollo como nación". Ver La personalidad histórica de Colombia y otros ensayos (Bogotá: Colcultura, 1977), 153.

${ }^{16}$ Ver el Volumen 1 de la Nueva historia de Colombia (Bogotá: Planeta, 1998), 234-237.

${ }^{17}$ James D. Henderson, "Recent Colombian Writing on Colombia", ponencia inédita presentada ante el South Eastern Council on Latin American Studies, marzo 7 de 2003. Ver también Elsy Marulanda, Colonización y guerras en el Sumapaz (Bogotá: CINEP, 1990); Alfredo Molano, Selva adentro: Una historia oral de la colonización del Guaviare (Bogotá: El Áncora Editores, 1992); Graciela Uribe Ramón, Veníamos con una manotada de ambiciones. Un aporte a la historia de la colonización del Caquetá (Bogotá: Editorial Universidad Nacional de Colombia, 1998). 


\section{El futuro}

El perfeccionamiento y la ampliación de la idea de "frontera" como construcción histórica, el profesionalismo mostrado por los estudios académicos de la historia y la cultura fronterizas y la incorporación de estos trabajos al grueso de la historiografía colombiana son tendencias presentes que, en mi opinión, seguirán aumentando y enriqueciendo nuestro entendimiento del pasado colombiano. En la segunda edición de su conmovedor testimonio autobiográfico Our Guerrillas, Our Sidewalks (Nuestras guerrillas, nuestras aceras), Herbert “Tico" Braun arguye que, mientras la Iglesia Católica y un fuerte espíritu nacionalista son el pegante que une a los colombianos, lo que más los divide es el conflicto entre las culturas urbana y rural. Braun recalca que, tras ser abandonados por sus líderes liberales en la década de los 50, los campesinos rebeldes que participaron en las etapas iniciales de La Violencia siguieron en la lucha. Éstos eran hombres de "pasiones al rojo vivo y ardorosas creencias", mientras que sus líderes eran "cautos y razonables".

Estos hombres de la ciudad — continúa Braun - tampoco querían acercarse demasiado a sus seguidores en el campo. No los consideraban sus iguales. Sus pasiones les sonaban tontas, exageradas [...]. En general se suponía, como se supone hoy, que la gente del campo había de perder la vida ${ }^{18}$.

Braun sostiene que, aún mientras la Nación avanza hacia el ideal de conformar una sociedad pluralista, "no existe prejuicio más profundo entre los colombianos que el que separa a la gente de la ciudad de la gente que vive en el campo, a los citadinos de los campesinos. Es un desprecio permanente que se siente en todas partes" ${ }^{\prime \prime}$.

En ninguna parte de Colombia predomina la población rural tanto como en los departamentos periféricos de las zonas fronterizas. A lo mejor, si el público urbano se ve expuesto a información realista acerca de la historia y las características únicas de las zonas fronterizas, podemos llegar a esperar que disminuya la patente división entre las culturas urbana y rural y con ella la violencia que por tanto tiempo ha azotado a esta nación.

\section{Bibliografía}

Academia del Meta, comp. Los Llanos: Una historia sin fronteras. Bogotá: Crear Arte, 1988. Angel de Flórez, Raquel. Conozcamos al Departamento del Meta. 2 vol. Bogotá: Talleres Gráficos del Fondo Rotatorio Judicial Penitenciaría Central, 1963.

\footnotetext{
${ }^{18}$ Herbert Braun, Our Guerrillas, Our Sidewalks: A Journey in to the Violence of Colombia (Rowman and Littlefield, 2003), 261.

${ }^{19}$ Ibídem, 266.
} 
Braun, Herbert. Our Guerrillas, Our Sidewalks: A Journey in to the Violence of Colombia (Rowman and Littlefield, 2003).

Cobos, María Teresa. "Guía bibliográfica para los Llanos Orientales de Colombia". Boletín Cultural y Bibliográfico 8, no. 12 (1965): 1888-1935.

Goulet Richard J. "Trade and Conversion: Indians, Franciscans and Spaniards on the Upper Amazon Frontier, 1693-1790". Tesis presentada para obtener el título de doctor. Massachusetts: University of Massachusetts-Amherst, 2003.

Henderson, James D. "Recent Colombian Writing on Colombia". Ponencia inédita presentada ante el South Eastern Council on Latin American Studies, marzo 7 de 2003.

Jaramillo, Jaime. La personalidad histórica de Colombia y otros ensayos. Bogotá: Colcultura, 1977).

Marulanda, Elsy. Colonización y guerras en el Sumapaz. Bogotá: CINEP, 1990.

Molano, Alfredo. Selva adentro: Una historia oral de la colonización del Guaviare. Bogotá: El Áncora Editores, 1992.

Nueva historia de Colombia. Vol. 1. Bogotá: Planeta, 1998.

Ortega Ricaurte, E. Villavicencio (1842-1942): Monografía histórica. Bogotá, 1943.

Pacheco, Juan M. Los jesuitas en Colombia. 2 vol. Bogotá: Editorial "San Juan Eudes", 19591962.

Pérez, Héctor. La participación de Casanare en la Guerra de Independencia 1809-1819. Bogotá: ABC, 1988.

Ramírez, Guillermo. "San Luis de Palenque: El llanero y su presente". Económica Colombiana, no. 2 (1954): 21-38.

Rausch, Jane M. A Tropical Plains Frontier: The Llanos of Colombia: 1531-1831. Albuquerque: University of New Mexico Press, 1984.

Reichel-Dolmatoff, Gerardo. Colombia. Nueva York: Thames and Hudson, 1965.

Romero, María Eugenia. Desde el Orinoco hacia el siglo XXI: El hombre, la fauna y su medio. Bogotá: Fondo FEN, 1989.

Röthlisberger, Ernst. El Dorado. Bogotá: Banco de la República, 1963.

Sweet, David. "The Ibero-American Frontier Mission in Native American History". En The New Latin American Mission History. Erick Langer y Robert H. Jackson, ed. Lincoln y Londres: University of Nebraska Press, 1995.

Turner, Frederick J. "The Significance of the Frontier in American History", Annual Report of the American Historical Association, 1893. Washington, D.C.: Government Printing Office, 1894.

Uribe, Graciela. Veníamos con una manotada de ambiciones. Un aporte a la historia de la colonización del Caquetá. Bogotá: Editorial Universidad Nacional de Colombia, 1998.

Wyman, W. D. y C. B. Kroeber. The Frontier in Perspective. Madison: University of Wisconsin Press, 1957.

Fecha de recepción del artículo: 15 de agosto de 2003.

Fecha de aceptación: 22 de agosto de 2003. 